LEPREL1 protein in SSc and control fibroblasts and measure possible induction by profibrotic factors, and 4) measure the effect of LEPREL1 gene editing on triple helical collagen secretion by SSc disease fibroblasts.

Methods: DNA samples from SSc patients and controls were assayed by qPCR for CNVs within regulatory regions of 40 candidate genes. The lead candidate, LEPREL1 was studied further through tagging SNP analysis, of rs 7612998 , rs1447936, s1018343, s696065, in 564 SSc and 627 controls. In wild type (WT), GPVI -/-single knockout (SKO) or LEPREL1-/- GPVI -/-double knockout (DKO) mice skin fibrosis was initiated by daily subcutaneous bleomycin for 14 days (LEPREL1-/- embryonically lethal due to placental thrombosis). Skin fibrotic lesions sampled on day 21 were analysed by histology and picrosirius red stain. SSc and control skin fibroblasts grown from forearm skin biopsies, were cultured at passage 3-5, with or without the pro-fibrotic TGFbeta, or with or without estradiol in cells grown in oestrogen depleted conditons (no phenol red). LEPREL1 was assayed by Western blot. CRISPR/Cas9 was used to edit the LEPREL1 gene in SSc skin fibroblasts.

Results: Initial screening of candidate genes revealed a weak statistical association between a first intron CNV nsv514192 and SSc susceptibility in males $(p<0.028)$. However SNP analysis demonstrated that a haplotype, identified as CTAA across the 4 SNPs, was associated with increased risk of SSc development (OR $3.45, p<0.0023)$. This allele opens a FOXA1 site in the first intron and was seen predominantly in females. In cultured dermal fibroblasts LEPREL1 protein levels were raised in SSc samples and induced to SSc levels in control fibroblasts by culture with TGFbeta. Estradiol also induced LEPREL1 and collagen I protein. In genetically modified LEPREL 1 knockout mice, resistance to bleomycin skin fibrosis was seen (figure 1). Silencing of LEPREL1 in SSc fibroblasts by CRISPR/ Cas9 preferentially reduced triple helical collagen I secretion.

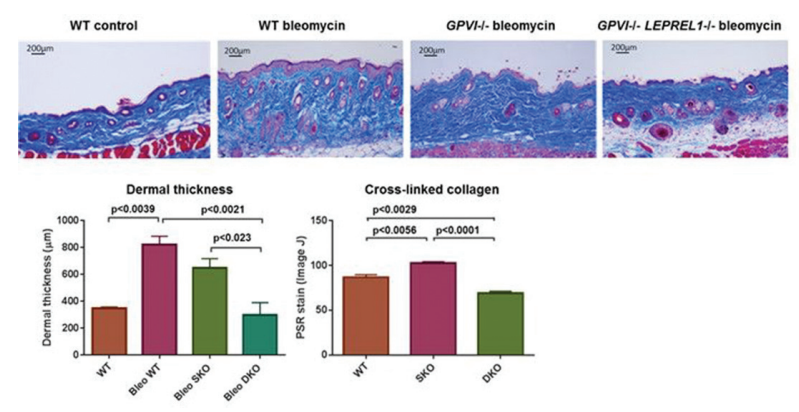

Conclusions: The prolyl 3-hydroxylase LEPREL1 is confirmed as a fibrosisrelated gene associated with SSc susceptibility. Small molecule inhibitors of this endoplasmic reticulum enzyme may limit the overproduction of collagen as a therapy for fibrosis.

Disclosure of Interest: None declared

DOI: 10.1136/annrheumdis-2018-eular.5513

\section{FRI0423 EFFECTS OF SELEXIPAG IN CULTURED SCLERODERMA SKIN FIBROBLASTS}

M. Cutolo ${ }^{1}$, B. Ruaro ${ }^{1}$, P. Montagna ${ }^{1}$, R. Brizzolara ${ }^{1}$, A.C. Trombetta ${ }^{1}$, S. Scabini ${ }^{2}$,

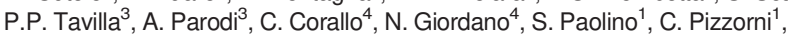
A. Sulli ${ }^{1}$, V. Smith ${ }^{5}$, S. Soldano ${ }^{1} .{ }^{1}$ Research Laboratory and Academic Division of Clinical Rheumatology, Department of Internal Medicine, University of Genoa, Polyclinic San Martino Hospital; ${ }^{2}$ Oncologic Surgery, Department of Surgery, Polyclinic San Martino Hospital; ${ }^{3}$ Department of Health Science, Unit of Dermatology, University of Genoa, Genoa; ${ }^{4}$ Department of Medicine, Surgery and Neurosciences, Scleroderma Unit, University of Siena, Siena, Italy; ${ }^{5}$ Department of Rheumatology, Ghent University Hospital, Ghent, Belgium

Background: In systemic sclerosis (SSc), the transition of fibroblasts into profibrotic myofibroblasts contributes to fibrosis through their over-production of extracellular matrix (ECM) proteins, primarily type I collagen (COL-1) and fibronectin (FN), a process which is mediated by the activation of fibrogenic intracellular signalling transduction molecules, including Erk1/2 and Akt kinases. ${ }^{1,2}$

Selexipag and its active metabolite (ACT-333679) are prostacyclin receptor agonists which leads to vasodilation in the pulmonary circulation and mainly used in the treatment of pulmonary arterial hypertension.

Objectives: To investigate the possible effects of selexipag and ACT-333679 in downregulating the profibrotic activity of cultured SSc fibroblasts/myofibroblasts and the fibrogenic signalling molecules involved in the process.

Methods: After obtaining Ethics Committee (EC) approval and informed consent, fibroblasts isolated from skin biopsies of 6 SSc patients (mean age $63 \pm 13$ years) were cultured until the 3rd culture passage and then either maintained in normal growth medium (untreated cells) or independently treated with different concentrations of selexipag (from $30 \mu \mathrm{M}$ to $0.3 \mu \mathrm{M}$ ) or ACT-333679 (from $10 \mu \mathrm{M}$ to $0.1 \mu \mathrm{M}$ ) for 48 hours. Protein and gene expressions of $\alpha$-smooth muscle actin $(\alpha-S M A)$, fibroblast specific protein-1 (S100A4), COL-1, and FN were investigated by Western blotting and qRT-PCR.

Erk1/2 and Akt phosphorylation was investigated in untreated and ACT-333679 treated cells at 15 and 30 min as well as at 48 hours by Western botting.

Results: Selexipag and ACT-333679 significantly reduced the protein synthesis and the gene expression of $\alpha$-SMA, S100A4, and COL- 1 in cultured SSc fibroblasts/myofibroblasts compared to untreated cells, primarily at the concentrations of $3 \mu \mathrm{M}$ and $0.3 \mu \mathrm{M}$ for selexipag and $1 \mu \mathrm{M}$ and $0.1 \mu \mathrm{M}$ for ACT-333679 $(p<0.05)$. Differently, FN was significantly downregulated at protein level $(p<0.05$ vs. untreated cells)

Of note, ACT-333679 significantly reduced the phosphorylation of Erk1/2 and Akt kinases already after $30 \mathrm{~min}$ of treatment in cultured SSc fibroblasts/myofibroblasts, and this effect was further maintained at 48 hours from treatment ( $p<0.05$ vs. untreated cells, for all tested concentrations).

Conclusions: For the first time selexipag and mainly its active metabolite ACT333679 were found to interfere with the profibrotic activity of cultured SSc fibroblasts/myofibroblasts, possibly through the downregulation of fibrogenic Erk $1 / 2$ and Akt intracellular signalling molecules.

\section{REFERENCES :}

[1] Ho YY, et al. Nature 2014;10:390-402.

[2] Distler JHW, et al. Arthrit Rheumatol 2017;69:257-67.

[3] Gatfield J, et al. J Pharmacol Exp Ther. 2017;362:186-99.

Disclosure of Interest: M. Cutolo Grant/research support from: Actelion, BristolMayer Squibb, Celgene, B. Ruaro: None declared, P. Montagna: None declared, R. Brizzolara: None declared, A. Trombetta: None declared, S. Scabini: None declared, P. P. Tavilla: None declared, A. Parodi: None declared, C. Corallo: None declared, N. Giordano: None declared, S. Paolino: None declared, C. Piz zorni: None declared, A. Sulli: None declared, V. Smith: None declared, S. Soldano: None declared DOI: 10.1136/annrheumdis-2018-eular.5348

\section{FRI0424 COMPARATIVE ANALYSIS OF CLINICALLY AFFECTED AND UNAFFECTED SKIN BIOPSIES FROM SCLERODERMA PATIENTS BASED ON RNA- SEQUENCING}

Z. Makowska ${ }^{1}$, A. Buttgereit ${ }^{1}$, S. Babaei ${ }^{1}$, N. Limaye ${ }^{2}$, C. Galant ${ }^{2}$, F. Houssiau $^{2}$, B. Weiss ${ }^{1}$, R. Lesche ${ }^{1}$, F. McDonald ${ }^{1}$, B. Lauwerys ${ }^{2} .{ }^{1}$ Bayer AG, Berlin, Germany; ${ }^{2}$ Université Catholique de Louvain, Brussels, Belgium

Background: Systemic sclerosis (SSc) is a complex chronic autoimmune disease characterised by vasculopathy, inflammation and fibrosis across different organ systems. The origin and pathogenesis of the disease are not well understood and symptoms may vary among affected individuals, complicating the diag nosis and therapy.

Objectives: We aimed at characterising gene expression profiles in biopsies from affected and unaffected skin of SSc patients.

Methods: RNA isolated from affected and unaffected skin biopsies of 23 SSc patients was analysed using polyA-selected RNA sequencing. Paired differentia expression analysis was performed with DESeq2, and Molecular Signatures Database gene set collections were used for functional annotation.

Results: Paired analysis of SSc gene expression profiles identified over 900 genes differentially regulated between the affected and unaffected skin biopsy samples (adjusted $\mathrm{p}$ value $<0.05$ ). Macroscopically unaffected skin samples showed clear gene expression differences from the affected skin samples, even though the histological examination showed that pathological changes were already present in most of the samples classified as unaffected. Clustering of differentially expressed genes and subsequent functional characterisation of the resulting gene modules revealed sets of co-expressed genes involved in inflammatory response, extracellular matrix interaction, metabolic function and epithelial differentiation, and highlighted the importance of a number of transcription drivers, such as serum response factor (SRF) or interferon regulatory factor 7 (IRF7). Several genes from the HOX family of transcription factors were found to be among the most significantly differentially expressed genes, pointing to an important role of wound healing and regeneration processes in SSc skin lesions. Additionally, we found significant association between expression of several proteasome subunits and the inflammation score obtained by histological assessment of the skin biopsies.

Conclusions: RNA sequencing analysis in paired skin biopsy samples revealed coordinated changes in the gene expression between SSc lesions and macroscopically unaffected skin, providing insights into the SSc skin pathogenesis at the molecular level. 